\title{
Perspective
}

\section{PTSD Treatment Problems at the U.S. Veterans Administration}

\author{
Steven G. Koven
}

check for

updates

Citation: Koven, S.G. PTSD Treatment Problems at the U.S. Veterans Administration. Psychiatry Int. 2021, 2, 25-31. https://doi.org/ $10.3390 /$ psychiatryint2010002

Received: 20 September 2020

Accepted: 5 January 2021

Published: 7 January 2021

Publisher's Note: MDPI stays neutral with regard to jurisdictional clai$\mathrm{ms}$ in published maps and institutional affiliations.

Copyright: $\odot 2021$ by the author. Licensee MDPI, Basel, Switzerland. This article is an open access article distributed under the terms and conditions of the Creative Commons Attribution (CC BY) license (https:// creativecommons.org/licenses/by/ $4.0 /)$.
Department of Urban and Public Affairs, University of Louisville, Louisville, KY 40208, USA; sgkove01@louisville.edu

\begin{abstract}
Post-traumatic stress disorder (PTSD) increasingly burdens the health of many people, as indicated by the growing numbers of U.S. military veterans' claims. An increased awareness of PTSD as a health concern necessitates a careful exploration of treatments. A range of current PTSD treatments and the need to more selectively utilize treatments is addressed. Studies indicate that recent PTSD treatment breakthroughs may offer promise and can be more effectively utilized. Assertions from organizations such as the U.S. National Center for PTSD contradict the commonly cited contention that there is no "cure" for PTSD. A review of PTSD literature reveals that: (1) the U.S. Veterans Administration is dispensing drugs (some of which have harmful side effects) that are not approved for use in treating PTSD by the U.S. Food and Drug Administration, (2) not aggressively exploring the use of novel approaches to PTSD treatment, and (3) not adequately funding research into PTSD treatment relatively to the funding of disability payments. Because of these factors the U.S. government is not optimally serving the interests of veterans.
\end{abstract}

Keywords: post-traumatic stress disorder; SGB; wilderness therapy; EMDR; NET

\section{Introduction}

Post-traumatic stress disorder (PTSD) exacts a high cost to society in terms of physical impairment, diminished productivity, and expenditure of government funds. Veteran payments are substantial and increasing [1]. By 2016, PTSD was the third most common disability veterans from all wars received compensation for and the most common mental health disorder experienced by those in combat [2]. In the U.S., PTSD claims are expanding rapidly. The numbers of veterans receiving U.S. Veterans Administration (VA) treatment for PTSD from the wars in Iraq and Afghanistan doubled between 2010 and 2016 [3]. In 2000, only nine percent of all veterans received disability benefits, yet by 2013, the proportion of all veterans receiving PTSD related benefits had risen to 16 percent. Adjusted for inflation to 2014 dollars, VA disability compensation to veterans amounted to $\$ 54$ billion in 2013 [4]. A Congressional Budget Office Study estimated that VA costs for first-year treatment for Iraq and Afghanistan veterans exceeded $\$ 2$ billion [5], and in 2017, the overall VA spending grew to $\$ 180$ billion [6]. Despite promising innovations in PTSD treatments and evidence of treatment efficacy, organizations such as the U.S. Veterans Affairs remain committed to status quo approaches. Such a mindset reduces the potential for more effective treatment. In light of the human and monetary costs of PTSD, it is incumbent upon researchers to carefully assess the available options. In addition to the monetary costs to taxpayers, PTSD exacts societal costs in terms of outcomes, such as poor marital relations, substance abuse, low self-esteem, and impaired sense of self-efficacy. Costs and benefits of approaches that focus on cognitive behavioral therapy, pharmaceutical medication, and nontraditional treatments are reviewed below. The possibility of adopting more innovative, safer, and more effective treatments is raised. 


\section{Literature Review}

\subsection{PTSD and Cognitive Behavioral Therapy}

Numerous treatments purport to be effective in reducing or eliminating symptoms that accompany PTSD. Research indicates that cognitive behavioral therapy (CBT) such as prolonged exposure therapy (PE) can be highly efficacious in treating PTSD across a wide range of trauma types, survivor characteristics, and cultures [7]. Advocates of PE contend that therapists without a great deal of prior experience can readily learn and implement the treatment successfully [8]. PE is said to be effective in removing the cues of past trauma and reduce feelings of anxiety. Another CBT treatment, cognitive processing therapy (CPT), has successfully treated a range of traumatic events, including military-related traumas [9]. Cognitive processing therapy is alleged to allow patients to restructure thoughts, which permits them to challenge prior interpretations that are commonly termed stuck points. Ultimately, this treatment permits patients to gain a healthier perspective about specific trauma they have experienced.

The cognitive behavioral therapy treatment of eye movement desensitization and reprocessing (EMDR) represents a third treatment type that has demonstrated effectiveness in treating PTSD [10]. The American Psychiatric Association (APA) notes that EMDR is effective in treating symptoms of acute and chronic PTSD and concludes that EMDR may be particularly useful for people who have trouble talking about the traumatic events they have experienced. The Department of Veterans Affairs and the Department of Defense recommend EDMR for the treatment of PTSD in both military and nonmilitary populations. The EMDR process, however, is controversial among healthcare professionals since it does not rely on "talk therapy" or medications but uses a patient's own rapid, rhythmic eye movements, which dampens the power of emotionally charged memories.

Narrative exposure therapy (NET) represents yet another CBT approach to addressing the problems associated with PTSD. In NET, patients are encouraged to talk about a traumatic event they have experienced. During therapy sessions, the severity of posttraumatic stress disorder is assessed; patients are then asked to depict their own life's happy and unhappy events. After this exercise, patients prioritize events and describe hopes for the future. An objective of NET is to have patients recount unhappy events and get acclimated toward those memories. In NET, talking about traumatic memories bridges "hot" memories (sensory details, emotions, and physiological reactions to trauma) and "cold" aspects of the trauma, such as place, date, time of day, and the people that were present at the time of the trauma. Narrative exposure therapy is intended to be quick and effective. In theory, by the end of the therapy, patients reconcile events of the past and acquire a better outlook for the future [11]. One advantage of narrative exposure therapy is its lower need for professional therapists than other types of assistance.

Narrative exposure therapy is especially useful in places where sufficient numbers of university-educated therapists are not present. The short duration of treatment and its limited costs are an important byproduct of this technique [12]. NET, however, remains controversial. Some authors contend that there are insufficient clinical and empirical studies to validate assertions of the treatment's advocates [13]. The Veterans Administration and Department of Defense recommend cognitive behavioral therapies such as CPT, PE, and EMDR as the most effective treatments for PTSD.

\subsection{PTSD and Pharmaceutical Medications}

Pharmaceutical medications are a common treatment modality for PTSD [14]. The selective serotonin reuptake inhibitor (SSRI) medications sertraline (Zoloft) and paroxetine (Paxil) represent two treatment drugs that are approved by the Food and Drug Administration (FDA) for relieving symptoms of PTSD. According to research trials, these drugs have demonstrated well-defined efficacy [15]. Research indicates that some SSRIs can achieve complete remission of PTSD symptoms in 20 to 30 percent of patients [16]. However, in 2004, the U.S. Food and Drug Administration issued a "Black Box" warning on SSRIs. The warning mentions the risk of suicidal thoughts, hostility, and agitation [17]. It must 
be noted that the FDA's approval is limited to only two SSRI medications (Zoloft and Paxil). Despite the absence of approval by the Food and Drug Administration (FDA), other antidepression medications are also prescribed by Veterans Administration physicians. These other medications include the serotonin-norepinephrine reuptake inhibitor (SNRI) Effexor and the selective serotonin reuptake inhibitor (SSRI) Prozac. These other drugs are sometimes used when more highly recommended medications (such as Zoloft and Paxil) are ineffective, unavailable, or not tolerated, even though the alternatives have not been approved by the FDA. The Veterans Administration concludes that medications rarely eliminate symptoms entirely but can provide symptom reduction. Physicians at the Department of Veterans Affairs have also prescribed benzodiazepine tranquilizers (such as Valium and Xanax) to veterans who have been diagnosed with PTSD. These tranquilizers are commonly dispensed; however, VA guidelines advise against their use for PTSD. The Veterans Administration dispenses these drugs to almost a third of veterans being treated for PTSD [18]. Physicians encourage caution in benzodiazepine/tranquilizer use; however, the VA continues their widespread use. Research is not supportive of a high degree of patient improvement with such treatment. One meta-analysis of the impact of benzodiazepines on PTSD patients indicates that benzodiazepine/tranquilizer drugs were not associated with improvements in PTSD-related outcomes and that use in patients with recent trauma did not decrease but rather increased the risk of developing PTSD [19]. Krystal et al. (2017) link a "PTSD pharmacological crisis" to three factors. First, only two drugs are currently approved by the FDA for PTSD treatment, and these drugs have been shown to only produce symptom reductions rather than remission of symptoms. Second, off-label drugs that have not been adequately studied are used for treatment of PTSD. Third, research and development of new medications for PTSD treatment has stalled. Paradoxically, progress has been made in the neuroscience of stress that could help to identify novel therapeutic targets [20].

A few clinical investigations have found that patients who experience hyper-arousal symptoms of PTSD could benefit from the stress-reducing capacity of glucocorticoids, commonly known as hydrocortisone or cortisone. Glucocorticoids enhance patients' coping abilities by facilitating information processing to diminish retrieval of fear-evoking memories. Research indicates that cortisol can diminish inappropriate retrieval of frightening memories and improve social functioning; however, since hydrocortisone is not FDA-approved to treat PTSD, close monitoring is advised [21].

\subsection{PTSD and Nontraditional Treatments}

A cottage industry of PTSD healers seems to have arisen in response to the growing numbers of PTSD patients. An array of therapeutic approaches exists. In one study, it was found that placing an anesthetic agent on nerves in the back of a patient's neck (a procedure referred to as stellate ganglion block or SGB) can relieve the symptoms of PTSD. A proponent of this innovation, anesthesiologist Eugene Lipov, concluded that while other PTSD treatments can take months or years to work with success rates of under 40 percent overall, success rates of the SGB treatment averaged 70-75 percent over the first nine years of use [22]. In addition, SGB is purported to be well-tolerated, fast-acting, and inexpensive in providing prolonged relief. According to Lipov, SGB treatment offers great promise for patients with PTSD who include veterans, victims of sexual assault, first responders, victims of crime, and others. Several related studies support the utility of SGB treatment. Navale et al. (2014) evaluated all published articles on the use of stellate ganglion block (SGB) as an adjunctive therapy for treatment of PTSD. The authors note that most patients experienced rapid improvement after SGB. However, they caution that robust clinical trials are needed to determine SGB's treatment efficacy for PTSD. They also conclude that pharmacotherapy and psychotherapy are only moderately helpful, and more effective treatment options need to be sought [23]. Lipov and Ritchie (2015) conclude that SGB may not only improve the quality of life for millions of patients but also reduce the overall socioeconomic burdens of treating PTSD [24]. Mulvaney et al. (2014), in a study of 
166 active-duty service members with multiple combat deployments experiencing anxiety symptoms associated with post-traumatic stress disorder (PTSD), found over $70 \%$ of the patients treated had a clinically significant improvement which persisted beyond 3 to 6 months postprocedure [25].

One meta-analysis concludes that there is reason to be optimistic about including physical activity (PA) as a useful intervention for people with PTSD [26]. Meditation is also suggested as a means of addressing the symptoms of PTSD. A randomized controlled trial (RCT) of the effectiveness of meditation on treating PTSD among Vietnam veterans found that subjects who practiced meditation experienced significant improvement in symptoms of PTSD, anxiety, depression, and insomnia, as well as measures of quality of life, such as employment, family problems, and stress reactivity [27]. An additional treatment, "wilderness therapy" (also referred to as outdoor behavioral healthcare), has also been utilized to treat PTSD. This therapy allows patients to become immersed in unfamiliar surroundings such as the wilderness. Programs are licensed by a state agency and overseen by a licensed mental health professional [28]. Some studies advocate self-help or self-healing as an ameliorative to PTSD. Self-healing occurs when the mind is able to construct new meaning out of violent situations that continue to replay in the brain. The construction of the new meaning helps the traumatized person to cope with their emotions. Self-healing then occurs with either a restoration of pre-trauma perspectives or the creation of a new perspective [29].

\subsection{Competing Perspectives of PTSD}

PTSD remains both a medical issue and one of ideology. The actual legitimacy of PTSD as a malady remains controversial. This questioning revolves around those who view PTSD as a physical illness and skeptics who question its validity. According to the first view, after years of ignoring reality, the harmful effects of trauma has at last been recognized. Under this perspective, people exposed to overwhelming trauma have a common mental and physical response that is treatable with proper care. Skeptics, on the other hand, question whether medical interest groups have created a "victim culture" in patients that undermines a person's natural resilience in the face of adversity. These skeptics argue that the 1980 recognition of PTSD by the American Psychiatric Association (APA) in their third edition of the Diagnostic and Statistical Manual of Mental Disorders (DSM-III) classification scheme was based as much on political as on scientific concerns. Skeptics contend that changes in the formal designation of PTSD were at least in part a response to lobbying on the part of Vietnam veterans and their clinicians. Researchers speculate that PTSD was assigned to soldiers taking part in a morally problematic war [30], and the assignment was consistent with a "compensation culture" that developed after the war consistent with a desire for restitution [31]. Countering the "skeptical" view is the opinion of numerous psychiatrists who assert that PTSD is dissimilar from common responses to stress, with some cases indicating a distinct biological profile [32].

Horwitz notes that perceptions of PTSD have been transformed from a condition marked by weakness to a diagnosis that warrants compassion, sympathy, and reward [32]. He argues that the PTSD official designation in 1980 reflected a "culture of therapy" that arose in the United States in the final decades of the twentieth century. This perspective led to a broadening of the definition of trauma, expanded the pool of people prone to developing trauma, and called for sympathetic responses to victims of trauma. Horwitz argues that a healing-oriented response to PTSD replaced both stoic attitudes that led the afflicted to bear their wounds silently and the punitive response to trauma that prevailed in earlier times. He states that a new vocation of grief and trauma counselors became widely institutionalized in many educational, medical, governmental, and business entities. Trauma care developed its own professional societies and journals as the chief source of PTSD research. This perspective eventually gained widespread acceptance [33]. 


\section{Conclusions}

The growth of PTSD cases and assertions that there is no "cure" for PTSD are balanced by literature that purports to show that various treatments are effective in reducing or eliminating symptoms associated with PTSD. For example, the National Center for PTSD website asserts that: (1) for every 100 people with PTSD who receive a trauma-focused therapy (such as cognitive processing therapy), 53 will no longer have PTSD after about three months, (2) for every 100 people with PTSD who receive a trauma-focused therapy (such as eye movement desensitization and reprocessing and prolonged exposure), 53 will no longer have PTSD after about three months, and (3) for every 100 people with PTSD who receive SSRI/SNRI pharmaceuticals, 42 will no longer have PTSD after about three months. Patients may still have some PTSD symptoms, but according to the U.S. National Center for PTSD, in most cases, symptoms will be manageable and will not occur very often [34]. The contentions that there is no "cure" for PTSD are in fundamental contradiction to the assertions of the National Center for PTSD and clinical tests. However, other literature is not sanguine regarding treatment effects. One study found that post-treatment conditions for PTSD patients remained at or above clinical criteria for PTSD, and the majority of patients (approximately two-thirds receiving approved treatments) retained their PTSD diagnosis after treatment [35]. A recent review of clinical trials paints a sobering picture of the usefulness of first-line psychotherapies in treating active-duty military personnel and veterans with post-traumatic stress disorder (PTSD). The review concludes that well-established treatment modalities such as prolonged exposure therapy (PE) and cognitive processing therapy (CBT) show limited effectiveness for treating PTSD in active-duty military personnel and veterans [36]. A Veterans Administration internal PTSD Psychopharmacology Working Group concluded that limited evidence on the pharmacotherapy of PTSD is a major obstacle to the effective treatment of the disorder and that a serious knowledge gap related to the efficacy of medications impedes the effective treatment of PTSD [20].

From my perspective as a nonmedical professional and U.S. combat veteran, and following a cursory review of the literature, a number of issues stand out that deserve greater attention and remedial action. First, the VA is dispensing large numbers of drugs (some of which have serious side effects) that have not been approved by the FDA. Remediation of this situation calls for greater public attention, public pressure to reverse the situation, further evidence of the benefits of alternative treatments, and if more expensive alternatives are shown to have greater efficacy, more funding is necessary. Second, ignoring FDA guidelines indicates questionable disregard for the objective conclusions of the government agency responsible for assessing the safety of medically prescribed drugs. This can have serious negative consequences for veterans in treatment for PTSD. Third, various novel treatments (such as SGB therapy, meditation, wilderness therapy) are described in the literature. The efficacy of these approaches is not well established. However, given the mixed findings in regard to the long-term effectiveness of other approaches, novel approaches deserve greater implementation and examination of their effectiveness in treating PTSD. Fourth, a much higher priority appears to be given to dispensing approved and unapproved drugs than to experimenting with novel approaches that may have positive effects. Fifth, the consequences of long-term dependency on specific drugs may not be given adequate attention when considering the net benefits of different approaches. Finally, it appears that by dispensing drugs with questionable safety, by not fully examining alternative treatments, and by poorly funding research into treatment relative to the amount of funding given to disability payment, the government is not optimally serving the interests of veterans. Individuals diagnosed with PTSD deserve access to the best available medical treatments. The Department of Veterans Affairs can explore more innovative and safer treatments in order to implement more effective remedies.

Funding: This research received no external funding.

Institutional Review Board Statement: Not applicable for studies not involving humans or animals. 
Informed Consent Statement: Not applicable for studies not involving humans.

Data Availability Statement: No new data were created or analyzed in this study. Data sharing is not applicable to this article.

Conflicts of Interest: The author declares no conflict of interest.

\section{References}

1. Koven, S.G. PTSD and suicides among veterans-recent findings. Public Integr. 2017, 19, 500-512. [CrossRef]

2. Nuss, L. Increase in VA Disability Claims for PTSD. Disability Advisor. Available online: https://www.disabilityadvisor.com/vadisability-claims / (accessed on 6 September 2019).

3. Sisk, R. Some Vets with PTSD Are Scamming the VA: Testimony. Available online: www.military.com/daily-news / (accessed on 6 September 2019).

4. Congressional Budget Office. Veterans' Disability Compensation: Trends and Policy Options. Available online: https://www.cbo. gov/publication/45615 (accessed on 16 September 2019).

5. Congressional Budget Office. The Veterans Health Administration's Treatment of PTSD and Traumatic Brain Injury among Recent Combat Veterans. Available online: www.cbo.gov/sites/default/files/ (accessed on 6 September 2019).

6. Congressional Budget Office. Possible Higher Spending Paths for Veterans' Benefits. Available online: https://www.cbo.gov/ system/files/2018-12/54881VA_Spending_Paths_0.pdf (accessed on 19 August 2020).

7. Foa, E.; Gillihan, S.; Bryant, R. Challenges and successes in dissemination of evidence-based treatments for posttraumatic stress: Lessons learned from prolonged exposure therapy for PTSD. Psychol. Sci. 2013, 14, 65-111. [CrossRef] [PubMed]

8. Foa, E.; Hembree, E.; Rothbaum, B. Prolonged Exposure Therapy for PTSD: Emotional Processing of Traumatic Experiences; Oxford University Press: New York, NY, USA, 2007.

9. Resick, P.; Monson, C.; Chard, K. Cognitive Processing Therapy: Veteran/Military Version; Department of Veterans' Affairs: Washington, DC, USA, 2008.

10. Shapiro, F. EMDR, adaptive information processing, and case conceptualization. J. EMDR Pract. Res. 2007, 1, 68-87. [CrossRef]

11. Varathan, D. Narrative Exposure Therapy: What's Your Story? Cognifit. Available online: https://blog.cognifit.com/whats-storynarrative-exposure-therapy/ (accessed on 24 July 2019).

12. Gwozdziewycz, N.; Mehl-Madrona, L. Meta-analysis of the use of Narrative Exposure Therapy for the effects of trauma among refugee populations. Perm J. 2013, 17, 70-76. [PubMed]

13. Etchison, M.; Kleist, D.M. Review of narrative therapy: Research and review. Fam. J. 2000, 8, 61-67. [CrossRef]

14. Koven, S.G. Posttraumatic Stress Disorder Treatments and Policy Recommendations; Scholar Press: Riga, Latvia, 2019.

15. Alexander, W. Pharmacotherapy for post-traumatic stress disorder in combat veterans focus on antidepressants and atypical antipsychotic agents. PET 2012, 37, 32-38.

16. Berger, W.; Mendlowicz, M.V.; Marques-Portella, C.; Kinrys, G.; Fontenell, L.; Marmar, C.R.; Figueira, I. Pharmacologic alternatives to antidepressants in posttraumatic stress disorder. Prog. Neuropsychopharmacol. Biol. Psychiatry 2009, 33, 169-180. [CrossRef] [PubMed]

17. Harvard Health Publishing. What Are the Real Risks of Antidepressants? 19 March 2019. Available online: https://www.health. harvard.edu/mind-and-mood/what-are-the-real-risks-of-antidepressants (accessed on 27 July 2019).

18. Olson, W. Sedatives Used for PTSD Treatment Despite Warnings. Stars and Stripes. 2019. Available online: https://www.military. com/daily-news/2013/04/08/sedatives-used-for-ptsd-treatment-despite-warnings.html (accessed on 28 July 2019).

19. Guina, J.; Rossetter, S.R.; DeRhodes, B.J.; Nahhas, R.W.; Welton, R. Benzodiazepines for PTSD: A systematic review and meta-analysis. J. Psychiatr. Pract. 2015, 2, 281-303. [CrossRef] [PubMed]

20. Krystal, J.M.; Davis, L.L.; Neylan, T.C.; Rasking, M.A.; Schnurr, P.P.; Stein, M.B.; Vessicchio, J.; Shiner, B.; Gleason, T.D.; Huana, G.D. It is time to address the crisis in the pharmacotherapy of Posttraumatic Stress Disorder:A Consensus Statement of the PTSD Psychopharmacology Working Group. Biol. Psychiatry 2017, 82, e51-e59. [CrossRef] [PubMed]

21. Motiwala, F. Do glucocorticoids hold promise as a treatment for PTSD? Curr. Psychiatry 2013, 12, 59-60.

22. Lipov, E.G. Using Stellate Ganglion Block (SGB) to Treat Post-Traumatic Stress Disorder. 22 August 2019. Available online: https:/ / www.anxiety.org/stellate-ganglion-block-sgb-for-ptsd-research-update (accessed on 12 September 2019).

23. Navaie, M.; Keefe, M.S.; Hickey, A.H.; McLay, R.N.; Ritchie, E.C.; Abdi, S. Use of Stellate Ganglion Block for Refractory Post-Traumatic Stress Disorder: A Review of Published Cases. J. Anesth. Clin. Res. 2014, 5, 403. [CrossRef]

24. Lipov, E.; Ritchie, E.C. A review of the use of stellate ganglion block in the treatment of PTSD. Curr. Psychiatry Rep. 2015, 17, 599. [CrossRef] [PubMed]

25. Mulvaney, S.W.; Lynch, J.H.; Hickey, M.; Rahman-Rawlins, T.; Schroeder, M.; Kane, S.; Lipov, E. Stellate Ganglion Block used to treat symptoms associated with combat-related post-traumatic stress disorder: A case series of 166 patients. Mil. Med. 2014, 179, 1133-1140. [CrossRef] [PubMed]

26. Rosenbaum, S.; Vancampfort, D.; Steel, Z.; Newby, J.; Ward, P.B.; Stubbs, B. Physical activity in the treatment of Post-traumatic stress disorder: A systematic review and meta-analysis. Psychiatry Res. 2015, 230, 130-136. [CrossRef] [PubMed]

27. Brooks, J.S.; Scarano, T. Transcendental meditation in the treatment of post-Vietnam adjustment. J. Couns. Dev. 1985, 64, 212-215. [CrossRef] 
28. GoodTherapy. Available online: https://www.goodtherapy.org/learn-about-therapy/types/wilderness-therapy (accessed on 16 August 2019).

29. Mollica, R. Healing Invisible Wounds: Paths to Hope and Recovery in a Violent World; Harcourt, Inc.: Orlando, FL, USA, 2006.

30. Burkett, B.G.; Whitley, G. Stolen Valor: How the Vietnam Generation Was Robbed of Its Heroes and Its History; Verity Press: Dallas, TX, USA, 1998; p. 232.

31. Summerfield, D. The invention of post-traumatic stress disorder and the social usefulness of psychiatric category. BMJ 2001, 322, 95-98. [CrossRef] [PubMed]

32. Brewin, C. Posttraumatic Stress Disorder: Malady or Myth? Yale University Press: New Haven, CT, USA, $2004 ;$ p. 42.

33. Horwitz, A. PTSD: A Short History; Johns Hopkins University Press: Baltimore, MD, USA, 2018; pp. 184, 186.

34. U.S. Department of Veteran Affairs. PTSD: National Center for PTSD: Treatment Comparison Chart. Available online: https: //www.ptsd.va.gov/apps/decisionaid/compare.aspx (accessed on 19 September 2019).

35. Steenkamp, M.M.; Litz, B.T.; Hoge, C.W.; Marmar, C.R. Psychotherapy for military-related PTSD A review of randomized clinical trials. JAMA 2015, 314, 489-500. [CrossRef] [PubMed]

36. Steenkamp, M.M.; Litz, B.T.; Marmar, C.R. First-line psychotherapies for military-related PTSD. JAMA 2020, $323,656-657$. [CrossRef] [PubMed] 